\title{
BMJ Open What is the evidence for efficacy, effectiveness and safety of surgical interventions for plantar fasciopathy? Protocol for a systematic review
}

\author{
C Siân MacRae (D) , ${ }^{1,2}$ Andrew J Roche, ${ }^{3}$ Tim J Sinnett, ${ }^{3}$ Neil E O'Connell ${ }^{1}$
}

To cite: MacRae CS, Roche AJ, Sinnett TJ, et al. What is the evidence for efficacy, effectiveness and safety of surgical interventions for plantar fasciopathy? Protocol for a systematic review. BMJ Open 2019;9:e031407. doi:10.1136/ bmjopen-2019-031407

- Prepublication history and additional material for this paper are available online. To view these files, please visit the journal online (http://dx.doi org/10.1136/bmjopen-2019031407).

Received 02 May 2019 Revised 16 August 2019 Accepted 30 August 2019
Check for updates

(C) Author(s) (or their employer(s)) 2019. Re-use permitted under CC BY-NC. No commercial re-use. See rights and permissions. Published by BMJ.

${ }^{1}$ Department of Clinical Sciences, Brunel University London, Uxbridge, UK

${ }^{2}$ Therapy Services, Chelsea and Westminster Hospital NHS Foundation Trust, London, UK

${ }^{3}$ Foot and Ankle Unit, Chelsea and Westminster Hospital NHS Foundation Trust, London, UK

Correspondence to

Dr C Siân MacRae;

sian.macrae@brunel.ac.uk

\section{ABSTRACT}

Introduction Plantar fasciopathy (PF) is a degenerative condition of the plantar fascia, secondary to repetitive overloading. For the majority, PF is self-limiting with greater than $80 \%$ of those affected gaining complete resolution within 1 year. However, persistent symptoms develop in approximately $10 \%$ of cases. Clinical practice guidelines for first-line treatment of PF recommend conservative management. For people with persistent symptoms that have not resolved following a trial of 6-12 months of conservative management, surgery may be offered. However, to date there are no systematic reviews of the effectiveness of the various surgical procedures for PF. We aim to systematically review quantitative studies assessing the effectiveness of surgical interventions in the management of PF.

Methods and analysis We will search for all published and unpublished randomised clinical trials evaluating surgical interventions in the management of PF. Cochrane Central Register of Controlled Trials (CENTRAL) in the Cochrane Library, MEDLINE (OVID), EMBASE (OVID), Web of Science (ISI) and Google Scholar will be searched without restrictions on date or language of publication. Inclusion criteria will include people over 18 years, diagnosed by clinical examination with $\mathrm{PF}$, or with an alternative diagnostic label (eg, plantar fasciitis, plantar heel pain, plantar fasciosis). The primary outcomes are changes in pain severity/intensity for first-step pain, and incidence and nature of adverse events. Secondary outcomes include foot and ankle-related disability/function, healthrelated quality of life, cost-effectiveness, changes in other reported measures of pain (eg, overall pain) and medication use. Outcomes will be assessed (1) short term ( $\leq 3$ months after intervention), (2) medium term (>3 months to $\leq 6$ months after intervention) or (3) long term ( $>6$ months to $\leq 2$ years after treatment). All data extraction will be performed by at least two independent reviewers on the basis of a priori developed extraction form. Where adequate data are found meta-analysis will be used to combine the results of studies for all core comparisons and outcomes using random effects models. Overall certainty of the evidence for each outcome will be assessed using the Grading of Recommendations Assessment, Development and Evaluation approach. Ethics and dissemination This systematic review does not require ethical approval as primary data will not be collected. The results of the study will be published in
Strengths and limitations of this study

To the best of our knowledge, this study will be the first systematic review that comprehensively explores and compares the effectiveness of various surgical interventions in people with plantar fasciopathy.

- This review focuses only on randomised controlled trials (RCT) as these designs offer the most robust estimates of effectiveness. No language limitations have been set, ensuring that the review is as comprehensive as possible.

- The findings from this study may provide guidance to healthcare providers to select appropriate management options for patients with persistent plantar fasciopathy, which may ultimately lead to a reduction in healthcare costs and improved patient outcomes.

- We recognise that only including RCTs limits the ability of our review to fully evaluate safety and adverse events.

- Our ability to draw strong conclusions may be restricted by the volume and quality of the identified studies.

a peer-reviewed journal and presented at appropriate conferences.

PROSPER0 registration number CRD42019133563.

\section{INTRODUCTION}

Plantar fasciopathy $(\mathrm{PF})$ is a degenerative condition of the plantar fascia, secondary to repetitive overloading. $\mathrm{PF}$ is characterised by symptoms of pain during weight-bearing activities, confined to the insertion of the plantar fascia at the anteromedial aspect of the calcaneum. ${ }^{1}$ Diagnosis of PF is typically made through clinical examination, with common features including pain on first few steps on waking or after prolonged sitting; pain on palpation of the medial plantar aspect of the calcaneus or proximal plantar fascia; plantar 
heel pain on passive dorsiflexion of the ankle and/or toes; and pain that worsens as the day progresses. ${ }^{2}$

PF affects approximately $10 \%$ of adults during their lifetime $^{3}$ with peak incidence of PF occurring between the ages of 45 and 64 years. ${ }^{4}$ There is a paucity of high-quality evidence to support most proposed risk factors for PF. ${ }^{5}$ Populations at risk, supported by strong evidence, include people who are overweight or obese, ${ }^{46}$ or have calf tightness. ${ }^{7}$ Risk factors, supported by a weak evidence, include pes planus ${ }^{89}$ or pes cavus feet, ${ }^{10}$ long-distance runners ${ }^{5}$ and people with occupations requiring prolonged standing. ${ }^{311}$

For the majority, PF is self-limiting with greater than $80 \%$ of those affected gaining complete resolution within 1 year. ${ }^{12}{ }^{13}$ However, persistent symptoms develop in approximately $10 \%$ of cases with detrimental effects on health-related quality of life (HRQoL) ${ }^{14}$ Difficulty walking may affect a person's ability to maintain a healthy weight, exercise, work and has been linked to anxiety, stress and depression. ${ }^{15}$ Hence, determining effective treatment approaches for persistent PF is essential.

Clinical practice guidelines for first-line treatment of PF recommend conservative management. ${ }^{2}$ Although multiple conservative treatment options are available, such as gel heel pads, exercise and extracorporeal shock wave therapy, ${ }^{16}{ }^{17}$ long-term effectiveness for many is uncertain or minimal. Surgical procedures, such as plantar fasciotomy ${ }^{18}$ or gastrocnemius release, ${ }^{19}$ may be offered to people with persistent PF whose symptoms have not resolved following a trial of 6-12 months of conservative management. ${ }^{1720}$ However, to date there are no systematic reviews of the effectiveness of these various surgical procedures for $\mathrm{PF}$.

\section{Objectives}

The primary aim of this systematic review is to determine the effectiveness and safety of surgical interventions in adults with PF.

\section{METHODS AND ANALYSIS}

The following criteria will be used for selecting studies for this review:

\section{Types of studies}

Randomised clinical trials published in any language will be included. Studies published in a language other than English will be translated. Studies in which participants were not randomised to intervention groups will be excluded.

\section{Types of participants}

Studies involving adults, aged 18 years or older, diagnosed with $\mathrm{PF}$, or with an alternative diagnostic label for this condition (eg, plantar fasciitis, plantar heel pain, plantar fasciosis) will be included. Studies will be included regardless of whether radiological diagnostic imaging has been employed and regardless of symptom duration.

\section{Types of interventions}

Any surgical procedure delivered as either a stand-alone treatment compared with placebo, no treatment, usual care or another intervention, or varying surgical procedures compared with each other will be included. Trials of surgery combined with another intervention will only be included if the comparisons allow for the specific evaluation of the effect of the surgery (eg, surgery and rehabilitation vs rehabilitation only).

\section{Types of outcome measures}

\section{Primary outcomes}

The following primary outcome measures will be analysed where such data are available:

1. Changes in pain severity/intensity for first-step pain. Examples of outcomes for pain include: visual analogue scale (VAS), numerical rating scale (NRS), verbal rating scale or Likert scale. Pain intensity will be presented and analysed as change on a continuous scale or in a dichotomised format as the proportion of participants in each group who attained a predetermined threshold of improvement. For example, cut-points from which to interpret the likely clinical importance of (pooled) effect sizes will be judged according to criteria proposed in the Initiative on Methods, Measurement, and Pain Assessment in Clinical Trials (IMMPACT) consensus statement. ${ }^{21}$ Specifically, reductions in pain intensity compared with baseline will be judged as follows:
A. $<15 \%$-no important change.
B. $\geq 15 \%$-minimally important change.
C. $\geq 30 \%$-moderately important change.
D. $\geq 50 \%$ - substantially important change.

2. The incidence and nature of adverse events such as injury, infection, rupture of the plantar fascia, worsening of symptoms and repeat procedures.

\section{Secondary outcomes}

The following secondary outcome measures will be analysed where such data are available:

1. Foot and ankle-related disability or function as measured by validated clinician-report and self-report questionnaires/scales. Examples of outcomes for disability/function include: the American Orthopaedic Foot and Ankle Society Score, the Foot Functional Index, the Manchester-Oxford Foot Questionnaire and the Foot Health Status Questionnaire.

2. Changes in HRQoL using any validated tool. Examples of outcomes for HRQoL include the 36-item Short Form Health Survey and the EuroQol-5 Dimension.

3. Cost-effectiveness.

4. Changes in other reported measures of pain (eg, overall pain).

5. Medication use.

\section{Timing of assessment of outcomes}

Primary and secondary outcomes were classified as: (1) short term ( $\leq 3$ months after intervention), (2) medium 
term ( $>3$ months to $\leq 6$ months after intervention), or (3) long term ( $>6$ months to $\leq 2$ years after treatment). For all outcomes, the latest outcome data within each time category were used for analysis. For example, if a study reported 3 and 6 -week pain outcomes, only the 6 -week data will be used.

\section{Search methods for identification of studies \\ Electronic searches}

The following electronic databases were searched on 28 June 2019 from their inception using a combination of controlled vocabulary, that is, Medical Subject Headings and free-text terms to identify published articles:

- Cochrane Central Register of Controlled Trials (CENTRAL) in the Cochrane Library.

- MEDLINE (OVID).

- EMBASE (OVID).

- Web of Science (ISI).

- Google Scholar.

There will be no language restrictions. All database searches will be based on this strategy but adapted to individual databases as necessary. The search strategy for MEDLINE is summarised in the online supplementary appendix 1.

\section{Searching other resources}

We will search ClinicalTrials.gov (www.clinicaltrials.gov) and the WHO International Clinical Trials Registry Platform (http://apps.who.int/trialsearch/) for ongoing trials. In addition, reference lists of retrieved articles will be checked for additional studies. The list of included studies will be sent to content experts to help identify any additional relevant studies.

\section{Data collection and analysis \\ Selection of studies}

The titles and abstracts of potential trials identified by the search strategy will be independently assessed by two review authors (SM and NOC) for their eligibility. If the eligibility of a study is unclear from the title and abstract, the full paper will be assessed. Studies that do not match the inclusion criteria will be excluded. Disagreements between review authors regarding a study's inclusion will be resolved by discussion. A third reviewer (AR) will assess relevant studies if resolution and agreement cannot be reached and a majority decision will be made. Studies will not be anonymised prior to assessment.

\section{Data extraction and management}

Two reviewers (NOC and SM) will independently extract data from all included studies using a standardised and piloted data extraction form. Discrepancies and disagreements will be resolved by consensus. In cases where consensus cannot be achieved, the trial will be assessed by a third reviewer (AR) for arbitration and a majority decision will be made.

We will extract the following data from each study included in the review:

- Country of origin.
- Study design.

- Study population (including diagnosis, diagnostic criteria used, symptom duration, age range, gender split).

- Details of concomitant treatments that may affect outcome (medication, procedures, and so on; what was permitted in the protocol and data on what was used).

- Sample size-active and control/comparator groups.

- Attrition rates by group for each follow-up point.

- Intervention(s) (including surgery type, type of surgeon (eg, podiatric surgeon), orthopaedic consultant or registrar, surgical approach, method of anaesthesia).

- Rehabilitation after surgery: including postsurgical care, rehabilitation programme received.

- Type and details of comparator intervention, including content, delivery, duration and dose where appropriate.

- Outcomes (primary and secondary) and time points assessed (only for the comparisons of interest to this review).

- Adverse effects, incidence and nature at all time points.

- Industry or other financial sponsorship; author conflict of interest statements.

\section{Assessment of risk of bias in included studies}

Two authors (SM and NOC) will independently assess risk of bias for each study using the criteria outlined in the Cochrane Handbook for Systematic Reviews of Interventions, ${ }^{22}$ with any disagreements resolved by discussion. In cases where consensus cannot be achieved, the trial will be assessed by a third reviewer (AR) for arbitration and a majority decision will be made.

We will assess the following for each study:

Random sequence generation (checking for possible selection bias). We will assess the method used to generate the allocation sequence as: low risk of bias (any truly random process, eg, random number table; computer random number generator); unclear risk of bias (method used to generate sequence not clearly stated); high risk of bias (studies using a non-random process, eg, odd or even date of birth; hospital or clinic record number).

Allocation concealment (checking for possible selection bias). The method used to conceal allocation to group prior to assignment determines whether intervention allocation could have been foreseen in advance of, or during recruitment, or changed after assignment. We will assess the methods as: low risk of bias (eg, telephone or central randomisation; consecutively numbered sealed opaque envelopes); unclear risk of bias (method not clearly stated); high risk of bias (studies that do not conceal allocation, eg, open list).

Blinding of participants: low risk of bias (participants blinded to allocated intervention; and unlikely that blinding is broken); unclear risk of bias (insufficient information to permit judgement of low/high risk of 
bias); high risk of bias (patients not blinded to allocated intervention OR patients blinded to allocated intervention but it is likely that blinding may have been broken (and a given outcome is likely to be influenced by lack of blinding)) .

Blinding of care providers: low risk of bias (care provider blinded to allocated intervention; and unlikely that blinding is broken OR no/incomplete blinding but judged that a given outcome is unlikely to be influenced by lack of blinding); unclear risk of bias (insufficient information to permit judgement of low/high risk of bias); high risk of bias (care provider not blinded to allocated intervention and the two interventions clearly identifiable to the care provider as experimental and control OR care provider blinded to allocated intervention but likely that blinding may have been broken (and a given outcome is likely to be influenced by lack of blinding)).

Blinding of assessor: low risk of bias (outcome assessor (including patients with respect to self-report outcomes) blinded to patients allocated intervention; and unlikely that blinding is broken, or no/incomplete blinding but judged that a given outcome is unlikely to be influenced by lack of blinding); unclear risk of bias (insufficient information to permit judgement of low/high risk of bias); high risk of bias (outcome assessor (including patients with respect to self-report outcomes) unblinded to patients allocated intervention OR outcome assessor blinded to allocated intervention but likely that blinding may have been broken (and a given outcome is likely to be influenced by lack of blinding)).

\section{Incomplete outcome data (dropouts)}

We will first check for possible attrition bias by considering if participant dropout rate is appropriately described and acceptable. Low: if less than 20\% dropout and appears to be missing at random. Numbers given per group and reasons for dropout described. Unclear: if less than $20 \%$ but reasons not described and numbers per group not given. Unclear that data are missing at random. High: if over $20 \%$ even if imputed appropriately.

\section{Incomplete outcome data (protocol violations)}

We will separately consider if participants were analysed in the group to which they were allocated. Low: if analysed data in group to which they were originally assigned (be that with appropriately imputed data or an available case analysis). Unclear: insufficient information provided to determine if analysis was per protocol or intention to treat. High: if per-protocol analysis was used. Where available data were not analysed or participant's data were included in group they were not originally assigned to.

\section{Selective reporting}

We will assess whether studies are free of the suggestion of selective outcome reporting. Methods will be assessed as: low risk of bias (study protocol available and all prespecified outcomes of interest adequately reported. Study protocol not available but all expected outcomes of interest adequately reported. All primary outcomes numerically reported with point estimates and measures of variance for all time points); high risk of bias (incomplete reporting of prespecified outcomes. One or more primary outcomes are reported using measurements, analysis methods or subsets of data that were not prespecified. One or more reported primary outcomes were not prespecified. One or more outcomes of interest reported incompletely and cannot be entered into a meta-analysis. Results for a key outcome expected to have been reported excluded).

\section{Other sources of bias}

We will consider other risk factors such as whether trials were stopped early, differences between groups at baseline, timing of outcome assessment, control of cointerventions and author source of funding declarations.

\section{Measures of treatment effect}

The size of treatment effect on pain intensity, as measured with a VAS or NRS, will be expressed using the mean difference (where all studies used the same measurement scale) or the standardised mean difference (SMD) (where studies used different scales). Where we pool data from different scales for which the direction of interpretation varies we will normalise the direction of the scales to a common direction. In order to aid interpretation of the pooled effect size the SMD will be back-transformed to a $0-100 \mathrm{~mm}$ VAS format on the basis of the median SD from trials using a $0-100 \mathrm{~mm}$ VAS where possible.

Risk ratio and risk difference with 95\% CIs will be calculated for dichotomised outcome measures. The number needed to treat to benefit and the number needed to treat to harm will be calculated as an absolute measure of treatment effect wherever possible.

\section{Unit of analysis issues}

Where an included trial compares multiple treatment arms to the same control and those arms are included in the same meta-analysis, the number of participants in control treatment arm will be split between those treatment arms.

\section{Dealing with missing data}

Where insufficient data are presented in the study report to enter into a meta-analysis, we will contact study authors to request access to the missing data. Waiting time for authors to respond has been set a priori to 1 month, with a reminder email sent at 2 weeks.

\section{Assessment of heterogeneity}

We will not combine studies that compared surgery to no treatment/usual care with studies that compared surgery to sham/placebo in the same analysis. We will assess heterogeneity using the $\chi^{2}$ test to investigate the statistical significance of such heterogeneity, and the $\mathrm{I}^{2}$ statistic to estimate the amount of heterogeneity. Where significant heterogeneity $(\mathrm{p}<0.1)$ is present, we will explore subgroup analyses. 
Preplanned comparisons are described in the 'Subgroup analysis and investigation of heterogeneity' section.

\section{Assessment of reporting biases}

We plan to use funnel plots to visually explore the likelihood of reporting biases when there are at least 10 studies in a meta-analysis and included studies differ in size, and we will use Egger's test to detect possible small study bias.

\section{Data synthesis}

Pooling of results will be performed where adequate data exist using Review Manager (RevMan V.5.3, 2014). Meta-analyses of outcome data will be undertaken only from suitably homogeneous studies using a random effects model.

We will perform a separate meta-analysis for the following classes of surgery: plantar fasciotomy, gastrocnemius release at the following time points: short term $(\leq 3$ months after intervention), medium term ( $>3$ months to $\leq 6$ months after intervention) or long term ( $>6$ months to $\leq 2$ years after treatment). For each broad class of surgery we will conduct the following comparisons where adequate data are available:

- Surgery versus sham surgery.

- Surgery versus minimal care/waiting list/no treatment.

- Surgery versus non-surgical treatment.

For all analyses, the outcome of the risk of bias assessments for included studies will be explicitly and clearly presented in the reporting. Where inadequate data are found to support statistical pooling, narrative synthesis of the evidence will be conducted.

\section{Certainty of the evidence}

We will assess the overall certainty of the evidence for each outcome using the Grading of Recommendations Assessment, Development and Evaluation (GRADE) approach, as recommended in the Cochrane Handbook for Systematic Reviews of Interventions. ${ }^{22}$ Two review authors (SM and NOC) will independently rate the quality of the evidence for each planned comparison.

Factors that may decrease the certainty of the evidence are:

- Study design and risk of bias (downgraded if more than $25 \%$ of the participants are from studies with a high risk of bias).

- Inconsistency of results (downgraded if significant heterogeneity is present by visual inspection or if the $\mathrm{I}^{2}$ value was greater than $\left.50 \%\right)$.

- Indirectness (generalisability of the findings; downgraded if more than $50 \%$ of the participants are outside the target group).

- Imprecision (downgraded if fewer than 400 participants are included in the comparison for continuous data or there are fewer than 300 events for dichotomous data, ${ }^{23}$ and other factors, eg, reporting bias, publication bias).
We will consider single studies with fewer than 400 participants for continuous or dichotomous outcomes inconsistent and imprecise, providing 'low certainty-evidence', which could be downgraded to 'very low-certainty evidence' if there are further limitations on the certainty of evidence. We will downgrade the certainty of the evidence for a specific outcome by a level, according to the performance of the studies against these five factors and we will describe them as follows. If there are multiple serious limitations for one domain we will consider downgrading the certainty of evidence by two levels.

The GRADE system uses the following criteria for assigning grade of evidence:

- High certainty: we are very confident that the true effect lies close to that of the estimate of the effect.

- Moderate certainty: we are moderately confident in the effect estimate; the true effect is likely to be close to the estimate of effect, but there is a possibility that it is substantially different.

- Low certainty: our confidence in the effect estimate is limited; the true effect may be substantially different from the estimate of the effect.

- Very low certainty: we have very little confidence in the effect estimate; the true effect is likely to be substantially different from the estimate of effect.

\section{Subgroup analysis and investigation of heterogeneity}

Where substantial heterogeneity is found $\left(\mathrm{I}^{2}>50 \%\right.$, $\mathrm{p}<0.10)$ we will conduct subgroup analysis investigating the possible impact of the type of surgical intervention (eg, fasciotomy vs gastrocnemius release) or surgical approach (open vs endoscopic).

\section{Sensitivity analysis}

Where sufficient data are available we will conduct sensitivity analysis on risk of bias (investigating the effect of including/excluding studies rated at high risk of bias (on one or more criteria other than blinding of patients or care providers) from the analysis and the choice of meta-analysis model (investigating the impact of applying a fixed effects instead of a random effects model)).

\section{Protocol amendments}

If any amendments are deemed necessary to this protocol, they will be documented in PROSPERO and amendments will be clearly stated in the final published systematic review manuscript.

\section{Patient and public involvement}

Patients and the public were not involved in the design and development of this protocol. The findings of the review will be available to healthcare professionals, policymakers and the public.

\section{Ethics and dissemination}

Ethical approval is not required for this study. The findings of this study may assist clinicians and guideline developers in providing recommendation to improve outcomes for people with persistent plantar fasciopathy. The 
procedures and findings of the study will be conducted in accordance with the Preferred Reporting Items for Systematic Reviews and Meta-Analyses-compliant guidelines. The anticipated end date for the study is June 2020. We aim to disseminate the findings of our systematic review through publication in a peer-reviewed journal and presentation at appropriate conferences.

\section{DISCUSSION}

This review will summarise the quantitative evidence available regarding the effectiveness of surgical procedures in the management of PF. The findings will help better inform clinicians regarding best practice surgical treatment approaches for patients with persistent PF.

\section{Twitter C Siân MacRae @Sian_MacRae}

Contributors After conceptualising and designing of the study, CSM and NOC registered the protocol on the PROSPERO database. All authors critically revised the protocol and contributed to the drafting of the final manuscript. CSM, NOC, TJS and AJR will perform the data collection and analyses. All authors read and approved the final manuscript.

Funding The authors have not declared a specific grant for this research from any funding agency in the public, commercial or not-for-profit sectors.

Competing interests None declared.

Patient consent for publication Not required.

Provenance and peer review Not commissioned; externally peer reviewed.

Open access This is an open access article distributed in accordance with the Creative Commons Attribution Non Commercial (CC BY-NC 4.0) license, which permits others to distribute, remix, adapt, build upon this work non-commercially, and license their derivative works on different terms, provided the original work is properly cited, appropriate credit is given, any changes made indicated, and the use is non-commercial. See: http://creativecommons.org/licenses/by-nc/4.0/.

\section{ORCID iD}

C Siân MacRae http://orcid.org/0000-0002-2060-670X

\section{REFERENCES}

1 Orchard J. Plantar fasciitis. BMJ 2012;345:e6603.

2 Martin RL, Davenport TE, Reischl SF, et al. Heel pain-plantar fasciitis: revision 2014. J Orthop Sports Phys Ther 2014;44:A1-A33.
3 Riddle DL, Pulisic M, Pidcoe P, et al. Risk factors for plantar fasciitis: a matched case-control study. J Bone Joint Surg Am 2003;85:872-7.

4 Riddle DL, Schappert SM. Volume of ambulatory care visits and patterns of care for patients diagnosed with plantar fasciitis: a national study of medical doctors. Foot Ankle Int 2004;25:303-10.

5 Buchbinder R. Clinical practice. Plantar fasciitis. N Engl J Med 2004;350:2159-66.

6 van Leeuwen KDB, Rogers J, Winzenberg T, et al. Higher body mass index is associated with plantar fasciopathy/'plantar fasciitis': systematic review and meta-analysis of various clinical and imaging risk factors. Br J Sports Med 2016;50:972-81.

7 Dyck DD, Boyajian-O'Neill LA. Plantar fasciitis. Clin J Sport Med 2004;14:305-9.

8 Irving DB, Cook JL, Young MA, et al. Obesity and pronated foot type may increase the risk of chronic plantar heel pain: a matched casecontrol study. BMC Musculoskelet Disord 2007;8:41.

9 Lee SY, Hertel J, Lee SC. Rearfoot eversion has indirect effects on plantar fascia tension by changing the amount of arch collapse. Foot 2010;20:64-70.

10 La Porta GA, La Fata PC. Pathologic conditions of the plantar fascia. Clin Podiatr Med Surg 2005;22:1-9.

11 Irving DB, Cook JL, Menz HB. Factors associated with chronic plantar heel pain: a systematic review. J Sci Med Sport 2006;9:11-22.

12 Martin RL, Irrgang JJ, Conti SF. Outcome study of subjects with insertional plantar fasciitis. Foot Ankle Int 1998;19:803-11.

13 Wolgin M, Cook C, Graham C, et al. Conservative treatment of plantar heel pain: long-term follow-up. Foot Ankle Int 1994:15:97-102.

14 Irving DB, Cook JL, Young MA, et al. Impact of chronic plantar heel pain on health-related quality of life. J Am Podiatr Med Assoc 2008;98:283-9.

15 Craft LL, Perna FM. The benefits of exercise for the clinically depressed. Prim Care Companion J Clin Psychiatry 2004;6:104-11.

16 Landorf KB, Menz HB. Plantar heel pain and fasciitis. BMJ Clin Evid 2008;2008. [Epub ahead of print: 05 Feb 2008].

17 NICE. Plantar fasciitis: clinical knowledge summaries, 2015. Available: https://cks.nice.org.uk/plantar-fasciitis [Accessed $5 \mathrm{Apr}$ 2019].

18 Bazaz R, Ferkel RD. Results of endoscopic plantar fascia release. Foot Ankle Int 2007;28:549-56.

19 Abbassian A, Kohls-Gatzoulis J, Solan MC. Proximal medial gastrocnemius release in the treatment of recalcitrant plantar fasciitis. Foot Ankle Int 2012;33:14-19.

20 Neufeld SK, Cerrato R. Plantar fasciitis: evaluation and treatment. J Am Acad Orthop Surg 2008;16:338-46.

21 Dworkin RH, Turk DC, Wyrwich KW, et al. Interpreting the clinical importance of treatment outcomes in chronic pain clinical trials: IMMPACT recommendations. J Pain 2008;9:105-21.

22 Higgins JPT, Green S, eds. Cochrane Handbook for Systematic Reviews of Interventions Version 5.1.0 [updated March 2011]. The Cochrane Collaboration 2011, 2011. http://handbook.cochrane.org

23 Mueller PS, Montori VM, Bassler D, et al. Ethical issues in stopping randomized trials early because of apparent benefit. Ann Intern Med 2007;146:878-81. 